\title{
Impact of topology-related attributes from Local Binary Patterns on texture classification
}

\author{
Thanh Phuong Nguyen, Antoine Manzanera, and Walter G. Kropatsch \\ ENSTA-ParisTech, 828 bd des Maréchaux, 91762 Palaiseau CEDEX, France \\ PRIP group, Favoritenstrae 9/186-3, A-1040 Wien, Austria \\ \{thanh - phuong.nguyen, antoine.manzanera\}@ensta- \\ paristech.fr,krw@prip.tuwien.ac.at
}

\begin{abstract}
A general texture description model is proposed, using topology related attributes calculated from Local Binary Patterns (LBP). The proposed framework extends and generalises existing LBP-based descriptors like LBP-rotation invariant uniform patterns $\left(\mathrm{LBP}^{\text {riu }} 2\right)$, and Local Binary Count (LBC). Like them, it allows contrast and rotation invariant image description using more compact descriptors than classic LBP. However, its expressiveness, and then its discrimination capability, is higher, since it includes additional information, including the number of connected components. The impact of the different attributes on texture classification performance is assessed through a systematic comparative evaluation, performed on three texture datasets. The results validate the interest of the proposed approach, by showing that some combinations of attributes outperform state-of-the-art LBP-based texture descriptors.
\end{abstract}

Keywords: local binary pattern, local descriptor, texture classification

\section{Introduction}

Texture recognition is a very active research topic in computer vision and pattern recognition. One of the most popular approaches for texture classification is based on feature distribution using Local Binary Pattern (LBP), introduced in [1. Since the generalised work of Ojala et al. 2], LBP is widely considered as an efficient descriptor for capturing local properties of images. The decisive advantages of LBPs are their low computational cost and their invariance to monotonic changes of illumination. These good properties allow to successfully apply LBPs not only to texture recognition, but also to many other areas of computer vision.

In the wake of LBP's success, many authors have introduced variants of LBP descriptors 3 to improve the performance of classic LBP, or to better suit it to a specific problem. Many different aspects have been considered. For preprocessing step, Gabor filters 4] have been used for capturing more global information. Different neighbourhoods, such as elliptical neighbourhood [5], three or fourpatch approaches [6] have been employed to exploit anisotropic information. To 
address the issue of LBP instability on near constant image areas, the Local Ternary Patterns [7] use three values $\{-1,0,1\}$ in the encoding step. Multiscale or multi-structure approaches [8,9] are considered to represent information at larger scales. Liao 10 chooses the most frequent patterns to improve the recognition accuracy. Guo et al. [11] use a complementary component related to magnitude to improve the texture classification.

In this paper, we propose a generic approach to improve the discrimination power of LBP by considering different geometrical and topological attributes extracted from LBPs. The proposed framework extends and generalises several existing LBP variants, and is also compatible (and then can be combined) with most of the other variants.

The remaining of the paper is organised as follows. The next section presents related works. Section 3 introduces the proposed framework, based on a family of rotation invariant attributes extracted from LBP. Section 4 is an evaluation of the descriptors derived from our models, compound with state-of-the-art descriptors, for the texture classification task applied on three classic datasets.

\section{$2 \quad$ LBP and its rotation invariant forms}

Local Binary Patterns 2 were introduced by Ojala et al. as a contrast invariant, binary version of the texture unit to represent its spatial structure. The binary pattern is formed by comparing a pixel value with its surrounding neighbours. The LBP encoding can be defined as follows:

$$
\operatorname{LBP}_{P, R}=\sum_{p=0}^{P-1} s\left(g_{p}-g_{c}\right) \cdot 2^{p}, s(x)=\left\{\begin{array}{l}
1, x \geq 0 \\
0, \text { otherwise }
\end{array}\right.
$$

where $g_{c}$ represents the gray value of the centre pixel and $g_{p}(0 \leq p<P)$ denotes the gray value of the neighbour pixel on a circle of radius $R$, and $P$ is the total number of neighbours. The sample values can be calculated by interpolation.

The concept of circular neighbourhood allows to introduce the notions of uniform LBP, and also of rotation invariant LBP. A LBP is said uniform if the number of bit-transitions (0-1 and 1-0) in a circular scan of the pattern is at most 2 . In texture description based on uniform LBP (denoted $\mathrm{LBP}^{u 2}$ ), non uniform LBPs are considered irrelevant, and then discarded or put in a single class. The rotation invariant LBP is defined as follows: $\operatorname{LBP}_{P, R}^{r i}=\min _{0 \leq i<P}\left\{R O R\left(\operatorname{LBP}_{P, R}, i\right)\right\}$, where $R O R(x, i)$ corresponds to the right circular bit-wise shift of $i$ bits on $P$-bit number $x$. Very good texture classification results have been reported 2$]$ using rotation invariant uniform patterns ( $\left.\mathrm{LBP}^{\text {riu } 2}\right)$.

Zhao et al. 12 introduced Local Binary Count as a variant of LBP. It ignores the local binary structure of LBP by only counting the number of "1" in the pattern. Although they dramatically simplify the geometric structure, LBC features have been used with success for texture classification. 


\section{Core Texture Model}

\subsection{Topology related LBP attributes}

The local descriptors used by our texture model embed and generalise several rotation invariant descriptors, including uniform patterns and Local Binary Count. They are based on a family of numerical attributes that are calculated on the original LBP. Consider the support of $\operatorname{LBP}_{P, R}$ as a set of $P$ points on a circle, where 2 consecutive points are said adjacent (see Fig. 1). Topological information can then be extracted from the LBP using the connected components (circular runs) of $1 \mathrm{~s}$ in the pattern. We will consider the following attributes:

- Number of connected components of 1s (\#)

- Length of the largest run of 1s (M)

- Length of the smallest run of 1s (m)

- Total number of $1 \mathrm{~s}(\Sigma)$

All these attributes are rotation invariant. \# is a topological measure, whose importance in the characterisation of shape is attested by a number of works in digital topology, in particular in the detection of critical points in thinning algorithms 13. The uniform patterns correspond to $\#=1$ or 0 . $\mathrm{M}$ and $\mathrm{m}$ can be seen as extensions of the uniform pattern values to non uniform patterns. $\Sigma$ is equivalent to the Local Binary Count. Figure 1 illustrates a non-uniform binary pattern (10111010) of 8 bits; with $\#=3, \mathrm{M}=3, \mathrm{~m}=1$ and $\Sigma=5$.

These attributes are not independent; all configurations of values are not possible and must respect the following constraints:

1. $\mathrm{m} \leq \mathrm{M} \leq \Sigma$

2. $0 \leq \# \leq\lfloor P / 2\rfloor$

3. if $\#=0, \mathrm{~m}=\mathrm{M}=\Sigma=0$

4. if $\#=1,1 \leq \mathrm{m}=\mathrm{M}=\Sigma \leq P$

5. if $\#>1,1 \leq \mathrm{M} \leq P-2 \#+1$

6. if $\#>1,1 \leq \mathrm{m} \leq\lfloor P / \#\rfloor-1$

7. if $\#>1, \# \leq \Sigma \leq P-\#$

These properties imply that for a combination of two or more attributes, the number of different configurations is relatively small compared to $2^{P}$ (see also Table 1).

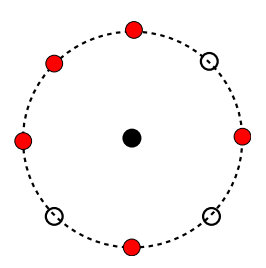

Fig. 1. A non-uniform pattern where 1 (resp. 0) is represented by red filled circle (resp. black circle).

\subsection{Texture modelling}

The purpose of this work is to evaluate the contribution of the different attributes in texture description. Every version of the descriptor used in the experiments is then related to a vector of $s$ attributes $\mathcal{A}=\left(\mathcal{A}_{1}, \ldots, \mathcal{A}_{s}\right)$. 
Basically, we describe a texture by computing, for each pixel, the LBP and its $s$ attributes, and then by calculating, for the whole image, the joint histogram of the $s$ attributes. The number of different attribute vectors depends on $P$, and on the chosen subset of attributes. In general, it is much smaller than $2^{P}$, the number of different LBPs.

In practice, to reduce the size of the histogram and the computation time of the descriptor, we associate a unique label to every value of the attribute vector, and pre-compute the label of all the LBP values in a label table $\Lambda$.

To do this, for every subset of $s$ attributes, we create an $s$-dimensional array $T$ initialized with zeros, and a scalar counter $c$ initialized to zero. Then we enumerate all the LBP values $n$ from 0 to $2^{P}-1$, and calculate the vector attribute $\mathcal{A}(n)$. If $T(\mathcal{A}(n))$ is equal to zero, we increment $c$, and set $T(\mathcal{A}(n))=c$. In all cases, we set the label table $\Lambda(n)=T(\mathcal{A}(n))$. The final value of the counter is denoted $N_{\mathcal{A}}$, the number of distinct vectors of attributes.

Finally we represent a texture by a histogram of labels:

$$
H(l)=\left|\left\{\mathbf{p} ; \Lambda\left(\operatorname{LBP}_{P, R}(\mathbf{p})\right)=l\right\}\right|
$$

In the experiments, we shall denote the texture descriptor based on the subset of attributes $\mathcal{A}$ as $\operatorname{LBP}_{P, R}^{\mathcal{A}}$, following the conventional notations $u 2$ or riu2 in LBP based models. Figure 2 shows a texture image with its corresponding label images and label histograms for the different configurations of $\mathrm{LBP}_{1,8}^{\mathcal{A}}$. In addition, Figure 3 shows images and histograms of labels corresponding to $\mathrm{LBP}_{1,8}^{\# M m}$ for different images, from the same texture class (first row), and from different classes (second row). The visual (di)similarity of histograms depending on the class is apparent on the figure.

To assess the interest of differentiating uniform patterns or not, a mixed texture representation $\left(\mathrm{LBP}_{P, R}^{r i u 2+\mathcal{A}}\right)$ is also evaluated in our work, by taking into account the above encoding only on non-uniform patterns, and using riu2 encoding for uniform patterns:

$$
\operatorname{LBP}_{P, R}^{r i u 2+\mathcal{A}}(\mathbf{p})=\left\{\begin{array}{l}
\operatorname{LBP}_{P, R}^{r i u 2}(\mathbf{p}), \text { if } \operatorname{LBP}_{P, R}(\mathbf{p}) \text { is uniform } \\
p+1+\Lambda\left(\operatorname{LBP}_{P, R}(\mathbf{p})\right), \text { otherwise }
\end{array}\right.
$$

\subsection{Relation with previous works}

As mentioned before, $\mathrm{LBP}_{P, R}^{\mathcal{A}}$ are related with other rotation invariant patterns: $\operatorname{LBP}_{P, R}^{r i u 2}[2]$ and LBC $[2]$. We now discuss further those relations.

- $\mathrm{LBP}_{P, R}^{\Sigma}$ is exactly LBC. It means that if $\Sigma \in \mathcal{A}, \mathrm{LBP}_{P, R}^{\mathcal{A}}$ is a generalisation of LBC.

- When $\operatorname{card}(\mathcal{A}) \geq 2$ and $(\# \in \mathcal{A}$ or $\Sigma \in \mathcal{A}), \mathrm{LBP}_{P, R}^{\mathcal{A}}$ is a superset of $\mathrm{LBP}_{P, R}^{\text {riu2 }}$ patterns. In that case indeed, riu 2 patterns are distinguished, either by the value of \# and anyone among $\{\mathrm{M}, \mathrm{m}, \Sigma\}$, or by one of the identity $\mathrm{M}=\Sigma$ or 
$\mathrm{m}=\left.\Sigma\right|^{1}$ Therefore, for such combination of attributes $\mathcal{A}, L B P_{P, R}^{\mathcal{A}}$ inherits the distinctive properties of $L B P_{P, R}^{\text {riu2 }}$, while containing more information. In this sense, $L B P_{P, R}^{\mathcal{A}}$ generalises $\mathrm{LBP}_{P, R}^{r i u 2}$.

- As a consequence, with the same conditions on $\mathcal{A}$, the performance of $\mathrm{LBP}_{P, R}^{\mathcal{A}}$ and $\mathrm{LBP}_{P, R}^{\text {riu } 2+\mathcal{A}}$ are the same.

- When $\operatorname{card}(\mathcal{A})=1$ or $\mathcal{A}=\{\mathrm{M}, \mathrm{m}\}, \mathcal{A}$ and riu2 are complementary, and $\mathrm{LBP}_{P, R}^{\text {riu } 2+\mathcal{A}}$ can be better than $\mathrm{LBP}_{P, R}^{\mathcal{A}}$ or $\mathrm{LBP}_{P, R}^{\text {riu2 }}$ alone.

Table 1 displays the number of labels (and then of histogram bins) for the different configurations of attributes. Note that the numbers for $\operatorname{LBP}_{P, R}^{\mathcal{A}}$ and $\mathrm{LBP}_{P, R}^{r i u 2+\mathcal{A}}$ are different only if $\operatorname{card}(\mathcal{A})=1$ or $\mathcal{A}=\{\mathrm{M}, \mathrm{m}\}$. In addition, Table 2 shows the number of labels for several existing LBP-based methods.

Table 1. Number of different labels, i.e. number of histogram bins of the texture descriptor in the different configurations.

\begin{tabular}{|l|c|c|c|c|c|c|c|c|c|c|c|c|c|c|c|}
\hline Schema & $\#$ & $\mathrm{M}$ & $\mathrm{m}$ & $\Sigma$ & $\mathrm{M} \#$ & $\mathrm{~m} \#$ & $\mathrm{M} \Sigma$ & $\mathrm{Mm}$ & $\# \Sigma$ & $\mathrm{m} \Sigma$ & $\mathrm{Mm} \#$ & $\# \mathrm{M} \Sigma$ & $\mathrm{Mm} \Sigma$ & $\# \mathrm{~m} \Sigma$ & $\# \mathrm{Mm} \Sigma$ \\
\hline $\mathrm{LBP}_{8,1}^{\mathcal{A}}$ & 5 & 9 & 9 & 9 & 18 & 14 & 21 & 15 & 18 & 18 & 22 & 23 & 23 & 22 & 23 \\
\hline $\mathrm{LBP}_{16,2}^{\mathcal{A}}$ & 9 & 17 & 17 & 17 & 66 & 36 & 92 & 59 & 66 & 66 & 125 & 180 & 159 & 125 & 212 \\
\hline $\mathrm{LBP}_{24,3}^{\mathcal{A}}$ & 13 & 25 & 25 & 25 & 146 & 62 & 225 & 135 & 146 & 146 & 353 & 680 & 557 & 353 & 989 \\
\hline $\mathrm{LBP}_{8,1}^{\text {riu }}+\mathcal{A}$ & 12 & 14 & 14 & 14 & 18 & 14 & 21 & 18 & 18 & 18 & 22 & 23 & 23 & 22 & 23 \\
\hline $\mathrm{LBP}_{16,2}^{\text {riu } 2+\mathcal{A}}$ & 24 & 30 & 30 & 30 & 66 & 36 & 92 & 66 & 66 & 66 & 125 & 180 & 159 & 125 & 212 \\
\hline $\mathrm{LBP}_{24,3}^{\text {riu }}+\mathcal{A}$ & 36 & 46 & 46 & 46 & 146 & 62 & 225 & 146 & 146 & 146 & 353 & 680 & 557 & 353 & 989 \\
\hline
\end{tabular}

Table 2. Number of different labels in several encodings.

\begin{tabular}{|l|c|c|c|}
\hline Method & $(\mathrm{P}, \mathrm{R})=(8,1)$ & $(\mathrm{P}, \mathrm{R})=(16,2)$ & $(\mathrm{P}, \mathrm{R})=(24,3)$ \\
\hline $\mathrm{LBP}_{P, R}^{\text {riu2 }}$ & 10 & 18 & 26 \\
\hline $\mathrm{LBP}_{P, R}^{u 2}$ & 59 & 243 & 555 \\
\hline $\mathrm{CLBP}_{P, R}^{\text {riu2 }}$ & 200 & 648 & 1352 \\
\hline
\end{tabular}

There is a strong link between $\operatorname{LBP}_{P, R}^{r i u 2+\mathcal{A}}$ with previous works aiming at exploiting information from non-uniform patterns to improve the texture descriptors. In particular $\operatorname{LBP}_{P, R}^{r i u 2+\{\Sigma\}}$ and $\operatorname{LBP}_{P, R}^{r i u 2+\{\#\}}$ are close to 14. In this work, the authors extended the notion of uniform pattern (as the $\Sigma$ attribute does), and the other patterns were encoded by the number of 0-1 transitions, which corresponds to the \# attribute.

\subsection{Completed texture descriptor}

Guo et al 11] proposed a state-of-the-art variant of LBP by coding the local differences as two complementary components: signs $\left(s_{p}=s\left(g_{p}-g_{c}\right)\right)$ and magnitudes $\left(m_{p}=\left|g_{p}-g_{c}\right|\right)$. They proposed to use two binary patterns, called

\footnotetext{
${ }^{1}$ Note that $\{\mathrm{M}, \mathrm{m}\}$ alone do not allow to distinguish uniform patterns, since the
} identity $\mathrm{M}=\mathrm{m}$ can occur with several connected components. 


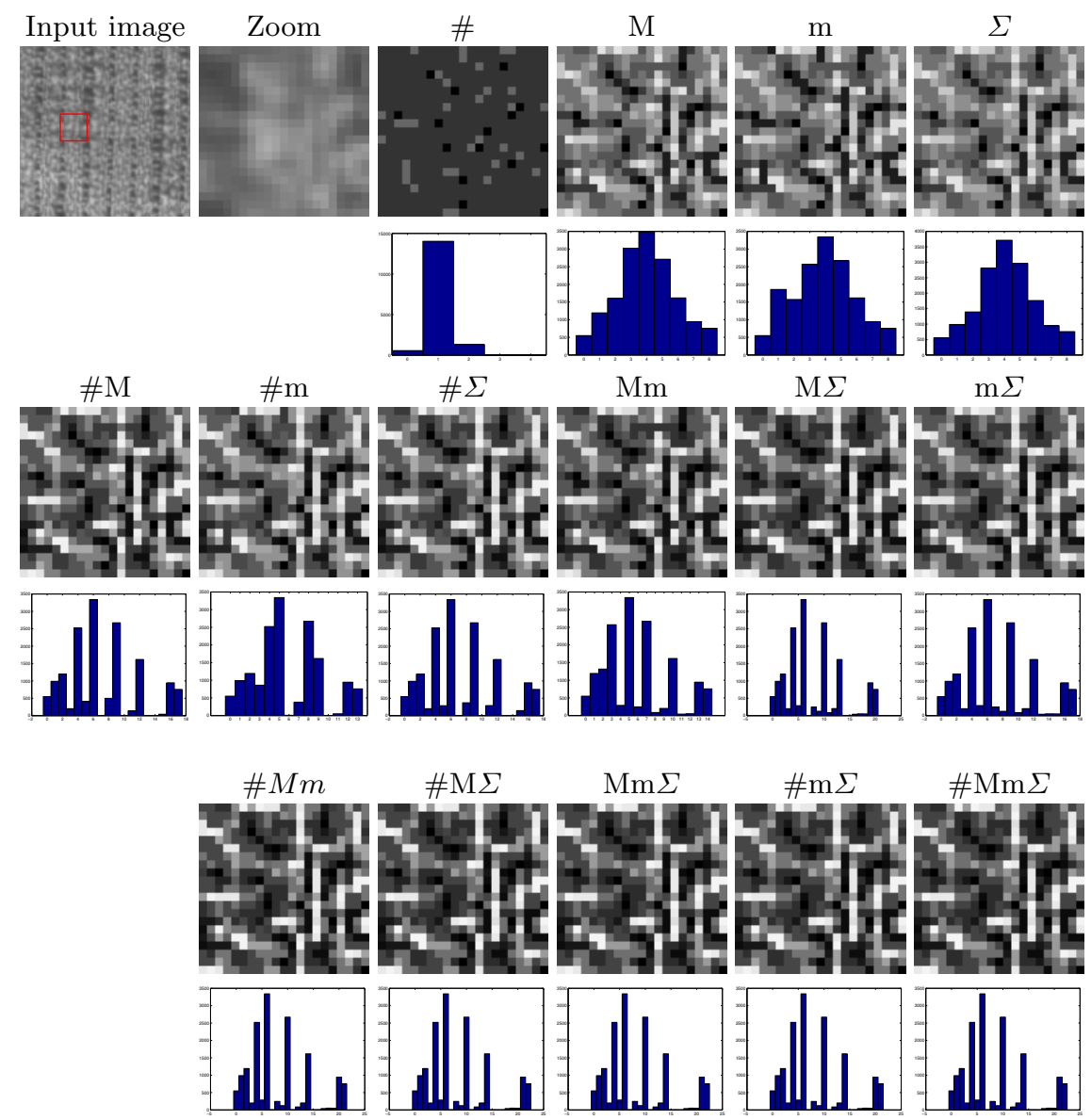

Fig. 2. A texture image and its label images and label histograms for the different configurations of attributes, with $(P, R)=(8,1)$. For the best visualization, the label images are zoomed from a part corresponding to the red square of the texture image.

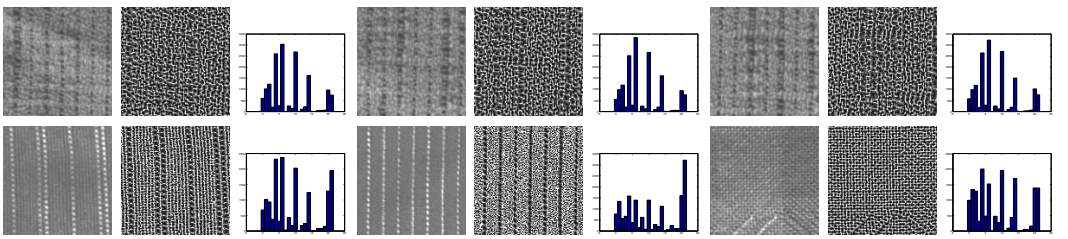

Fig. 3. Texture images and their label images and histograms for $\mathrm{LBP}_{8,1}^{\# M m}$. The first row contains images of the same class, the second row images of different classes. 
CLBP-Sign (CLBP_S) and CLBP-Magnitude (CLBP_M). The first pattern is identical to the LBP. The second one which measures the local variance of magnitude is defined as follows:

$$
\text { CLBP_M } \mathrm{M}_{P, R}=\sum_{p=0}^{P-1} s\left(m_{p}-\tilde{m}\right) \cdot 2^{p},
$$

where $\tilde{m}$ is the mean value of $m_{p}$ for the whole image. In addition, Guo et al. observed that the local value itself carries important information. Therefore, they defined the operator CLBP-Center (CLBP_C) as follows:

$$
\text { CLBP_C }=s\left(g_{c}-\tilde{g}\right),
$$

where $\tilde{g}$ is the mean gray level for the whole image. Because these operators are complementary, their combination leads to a significant improvement, and then CLBP is now considered a reference method in texture classification.

Inspired from this work, we also evaluated our descriptors by complementing the difference sign information (CLBP_S) by the difference magnitude (CLBP_M) and gray level (CLBP_C). For CLBP_S and CLBP_M, instead of using riu2 mapping, we apply our proposed encoding to obtain $\operatorname{CLBP}_{P, R}^{\mathcal{A}}$ and CLBP_M $\mathrm{M}_{P, R}^{\mathcal{A}}$. Finally, the feature vector of the whole image is constructed by considering the joint histograms of CLBP_S $\mathcal{A}_{P, R}^{\mathcal{A}}, \mathrm{CLBP}_{-} \mathrm{M}_{P, R}^{\mathcal{A}}$ and CLBP_C. Then, if LBP ${ }_{P, R}^{\mathcal{A}}$ has $n$ different labels, $\mathrm{CLBP}_{P, R}^{\mathcal{A}}$ has $2 n^{2}$ labels (see also Table 1 ).

\subsection{Texture classification}

Because the contribution of this work is focused on texture descriptors, and the competing LBP based methods all used $\chi^{2}$ distance as similarity metrics [2, and nearest neighbour as classification criterion, we used the same classification method for fair comparison purposes. If $H_{1}$ and $H_{2}$ are two attribute label histograms, the $\chi^{2}$-dissimilarity between the two textures is:

$$
\chi^{2}\left(H_{1}, H_{2}\right)=\sum_{i=1}^{N} \frac{\left(H_{1}(i)-H_{2}(i)\right)^{2}}{H_{1}(i)+H_{2}(i)},
$$

with $N=N_{\mathcal{A}}$ or $N=N_{\text {riu } 2+\mathcal{A}}$ the number of labels.

\section{Experiments}

\subsection{Datasets}

The effectiveness of the proposed method is assessed by a series of experiments on three large and representative databases: Outex [15, CUReT [16 and UIUC [17.

The Outex database (examples are shown in Figure 6) contains images captured from a wide variety of real materials. We consider the two commonly used test suites, Outex_TC_00010 (TC10) and Outex_TC_00012 (TC12), containing 
24 classes of textures. Each image may be seen under nine different rotation angles between 0 and $90^{\circ}$. For TC10, The texture images at angle $0^{\circ}$ are chosen for training the classifier, all the remaining images are used for testing. For TC12, aside from the different viewing angles, the images can have three types of illumination: "inca", used for learning, and "t184" or "horizon", used for testing (test sets are denoted TC12t and $\mathrm{TC} 12 \mathrm{~h}$ respectively).

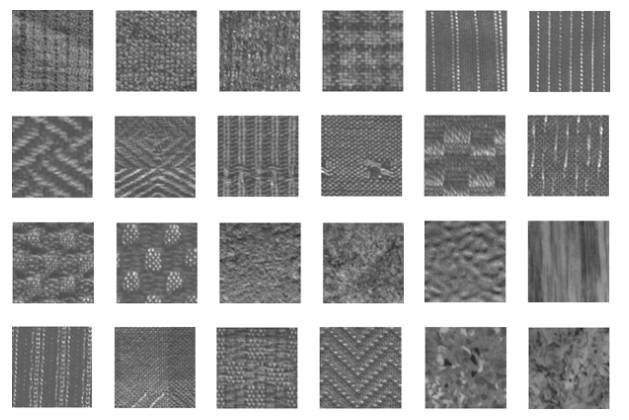

Fig. 4. Texture images with the illumination condition "inca" and zero degree rotation angle from the 24 classes of textures on the Outex database.

The CUReT database contains 61 texture classes (see Figure 5 a), each having 205 images acquired at different viewpoints and illumination orientations. We follow the experimental protocol proposed in [18, 19], using 4 different learning sets made of $6,12,23$ and 46 images (first line of Table 7).

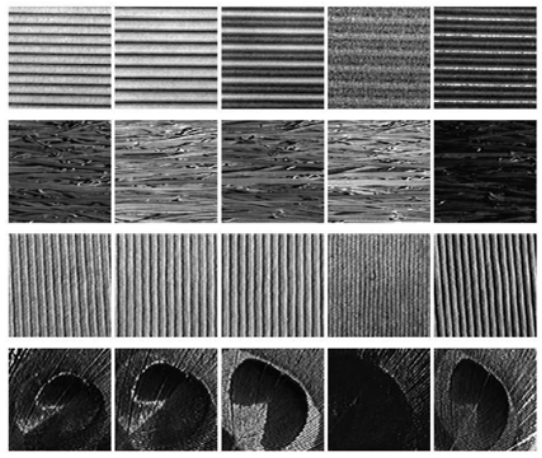

(a) CUReT dataset

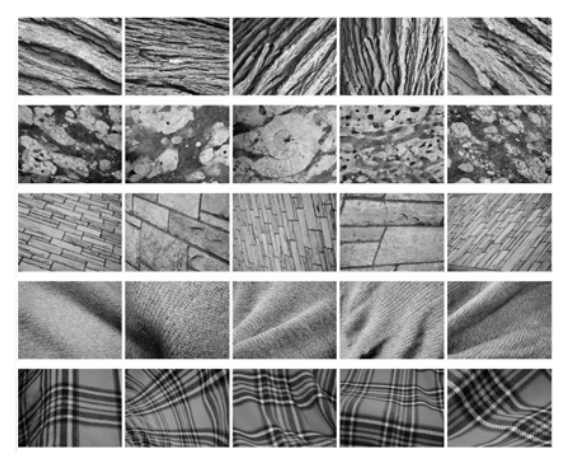

(b) UIUC dataset

Fig. 5. Examples of texture images.

The UIUC texture database includes 25 classes with 40 images in each class. The resolution of each image is $640 \times 480$. The database contains materials im- 
aged under significant viewpoint variations (examples are shown in Figure 5 b). Following 17], to eliminate the dependence of the results on the particular training images used, four different learning sets of 5, 10, 15 and 20 images are used while the remaining images per class are used as test set.

In the upcoming result sections, the performance measure will be given in percentage of correct classification. As the typical size of the test sets is around 5000 , the percentage values are rounded to the first decimal. Furthermore, our methods is practically deterministic (ignoring the slight influence of interpolation in the computation of the LBP). Finally, the test protocol of the Outex dataset is also deterministic, and the typical observed standard deviation in the cross validation schemes of Curet and UIUC is less than $0.1 \%$.

\subsection{Computational cost}

We consider in this section the computational cost of our descriptors with respect to other LBP-based operators. Experiments on Outex TC10 test suite containing 4320 images of $128 \times 128$ pixels were performed on a machine with $2.0 \mathrm{GHz} \mathrm{CPU}$, 4 Go RAM and Linux 3.2.0-23 kernel. Table 3 presents the computation time (in seconds) of different descriptors in the configuration $(P, R)=(2,16)$ and reports the total time (in seconds) for classifying the 3840 test images against the 480 reference images.

Table 3. Complexity of our different descriptors with respect to LBP ${ }^{\text {riu2 }}$ (FET: Feature Extraction Time, MT: Matching Time).

\begin{tabular}{c||c||c|c|c|c|c|c|c|c|c|c|c|c|c|c|c|c} 
Method & riu2 & $\#$ & $\mathrm{M}$ & $\mathrm{m}$ & $\Sigma$ & $\mathrm{M} \#$ & $\mathrm{~m} \#$ & $\mathrm{M} \Sigma$ & $\mathrm{Mm}$ & $\# \Sigma$ & $\mathrm{m} \Sigma$ & $\mathrm{Mm} \#$ & $\# \mathrm{M} \Sigma$ & $\mathrm{Mm} \Sigma$ & $\# \mathrm{~m} \Sigma$ & $\# \mathrm{Mm} \Sigma$ \\
\hline FET & 78.1 & 79.2 & 78.4 & 78.3 & 80.2 & 80.9 & 80.8 & 79.2 & 78.7 & 79.5 & 78.9 & 80.3 & 80.6 & 83.3 & 83.3 & 82.2 \\
\hline MT & 1.2 & 0.9 & 1.1 & 1.1 & 1.2 & 4.7 & 2.1 & 4.7 & 2.7 & 4.5 & 3.2 & 6.9 & 11.4 & 10.1 & 6.5 & 13.1
\end{tabular}

As can be seen from Table 3 , the descriptor construction time does not vary much from one method to the other, while the classification time is proportional to the length of the feature vector.

\subsection{1st experiment: $\operatorname{LBP}_{P, R}^{\mathcal{A}}$ and $\operatorname{LBP}_{P, R}^{\text {riu2+A }}$}

Table 4 compares our descriptors $\left(\operatorname{LBP}_{P, R}^{\mathcal{A}}\right)$ with the classic $\mathrm{LBP}_{P, R}^{\text {riu2 }}$ on Outex dataset, for different $(P, R)$ configurations. Those results can be interpreted as follows:

- The four attributes have distinct properties. Considered alone, their performance is comparable to $\mathrm{LBP}_{P, R}^{r i u 2}$, except for \#, whose expressiveness is too weak if taken alone.

- Jointly considering 2 attributes, the results are (except in one case) better than $\mathrm{LBP}_{P, R}^{\text {riu2 }}$, with an average improvement which can reach $6 \%$. 


\begin{tabular}{|c|c|c|c|c|c|c|c|c|c|c|c|c|}
\hline \multirow[t]{2}{*}{ Method } & \multicolumn{4}{|c|}{$(\mathrm{P}, \mathrm{R})=(8,1)$} & \multicolumn{4}{|c|}{$(\mathrm{P}, \mathrm{R})=(16,2)$} & \multicolumn{4}{|c|}{$(\mathrm{P}, \mathrm{R})=(24,3)$} \\
\hline & TC10 & TC12 t & TC12 h & Mean & TC10 & TC12 t & TC12 h & Mean & TC10 & $\mathrm{TC} 12 \mathrm{t}$ & TC12 h & Mean \\
\hline \begin{tabular}{|l|l}
$\mathrm{LBP}^{\text {riu2 }}$ & 2 \\
\end{tabular} & 84.8 & 65.5 & 63.7 & 71.3 & 89.4 & 82.3 & 75.2 & 82.3 & 95.1 & 85.0 & 80.8 & 87.0 \\
\hline $\mathrm{LBP}^{\#}$ & 52.4 & 37.4 & 32.0 & 40.6 & 66.5 & 51.3 & 47.1 & 55.0 & 77.0 & 67.5 & 58.2 & 67.6 \\
\hline Gain & - & - & - & - & - & - & - & - & - & - & - & - \\
\hline $\mathrm{LBP}^{M}$ & 83.6 & 67.1 & 64.4 & 71.7 & 87.6 & 82.2 & 78.9 & 82.9 & 95.9 & 88.1 & 86.4 & 90.1 \\
\hline Gain & - & 1.6 & 0.7 & 0.4 & - & - & 3.7 & 0.6 & 0.8 & 3.1 & 5.6 & 3.1 \\
\hline $\mathrm{LBP}^{m}$ & 84.8 & 64.5 & 62.2 & 69.9 & 91.0 & 82.9 & 77.0 & 83.7 & 96.5 & 86.2 & 80.4 & 87.7 \\
\hline Gain & 0.0 & - & - & - & 1.6 & 0.6 & 1.8 & 1.4 & 1.4 & 1.2 & - & 0.7 \\
\hline $\mathrm{LBP}^{2}$ & 82.9 & 65.0 & 63.2 & 70.4 & 88.7 & 82.6 & 77.4 & 82.9 & 91.3 & 83.8 & 82.7 & 86.0 \\
\hline Gain & - & - & - & - & - & 0.3 & 2.2 & 0.6 & - & - & 1.9 & - \\
\hline $\mathrm{LBP}^{M \#}$ & 86.5 & 69.9 & 66.2 & 74.2 & 93.7 & 85.3 & 82.1 & 87.1 & 96.8 & 88.7 & 84.3 & 89.9 \\
\hline Gain & 1.7 & 4.4 & 2.5 & 2.9 & 4.3 & 3.0 & 6.9 & 4.8 & 1.7 & 3.7 & 3.5 & 2.9 \\
\hline $\mathrm{LBP}^{m \#}$ & 85.7 & 67.4 & 66.4 & 73.1 & 93.1 & 85.9 & 81.4 & 86.8 & 97.5 & 89.3 & 85.0 & 90.6 \\
\hline Gain & 0.9 & 1.9 & 2.7 & 1.8 & 3.7 & 3.6 & 6.2 & 4.5 & 2.4 & 4.3 & 4.2 & 3.6 \\
\hline $\mathrm{LBP}^{M \nu}$ & 85.8 & 69.7 & 66.6 & 74.0 & 92.5 & 85.9 & 82.3 & 86.9 & 96.9 & 89.9 & 86.0 & 91.0 \\
\hline Gain & 1.0 & 4.2 & 2.9 & 2.7 & 3.1 & 3.6 & 7.1 & 4.6 & 1.8 & 4.9 & 5.2 & 4.0 \\
\hline $\mathrm{LBP}^{M m}$ & 85.6 & 66.8 & 63.6 & 72.0 & 92.5 & 85.4 & 82.4 & 86.8 & 98.1 & 92.2 & 87.2 & 92.5 \\
\hline Gain & 0.8 & 1.3 & - & 0.7 & 3.1 & 3.1 & 7.2 & 4.5 & 3.0 & 7.2 & 6.4 & 5.5 \\
\hline $\mathrm{LBP}^{\# \Sigma}$ & 87.1 & 69.8 & 67.8 & 74.9 & 93.4 & 84.6 & 79.7 & 85.9 & 96.5 & 87.5 & 83.6 & 89.2 \\
\hline Gain & 2.3 & 4.3 & 4.1 & 3.6 & 4 & 2.3 & 4.5 & 3.6 & 1.4 & 2.5 & 2.8 & 2.2 \\
\hline $\mathrm{LBP}^{m \Sigma}$ & 86.0 & 70.1 & 66.8 & 74.3 & 92.9 & 85.8 & 83.4 & 87.4 & 97.8 & 91.4 & 86.8 & 92.0 \\
\hline Gain & 1.2 & 4.6 & 3.1 & 3.0 & 3.5 & 3.6 & 8.2 & 5.1 & 2.7 & 6.4 & 6.0 & 5.0 \\
\hline $\mathrm{LBP}^{M m \#}$ & 85.8 & 70.5 & 68.2 & 74.8 & 94.3 & 86.8 & 84.2 & 88.4 & 97.2 & 90.9 & 86.7 & 91.6 \\
\hline Gain & 1.0 & 5.0 & 4.5 & 3.5 & 4.9 & 3.5 & 9.0 & 6.1 & 2.1 & 5.9 & 5.9 & 4.6 \\
\hline $\mathrm{LBP}^{\# M L}$ & 86.0 & 70.6 & 67.9 & 74.8 & 93.7 & 87.0 & 84.3 & 88.3 & 97.2 & 90.4 & 86.2 & 91.3 \\
\hline Gain & 1.2 & 5.1 & 4.2 & 3.5 & 4.3 & 4.7 & 9.1 & 6.0 & 2.1 & 5.4 & 5.4 & 4.3 \\
\hline $\mathrm{LBP}^{M m \Sigma}$ & 86.0 & 70.6 & 67.9 & 74.8 & 94.1 & 87.3 & 84.1 & 88.5 & 97.0 & 90.3 & 86.4 & 91.2 \\
\hline Gain & 1.2 & 5.1 & 4.2 & 3.5 & 4.7 & 5.0 & 8.9 & 6.2 & 1.9 & 5.3 & 5.6 & 4.2 \\
\hline $\mathrm{LBP}^{\# m \Sigma}$ & 86.1 & 70.8 & 67.8 & 74.9 & 94.1 & 87.0 & 84.1 & 88.4 & 97.4 & 91.0 & 86.7 & 91.7 \\
\hline Gain & 1.3 & 5.3 & 4.1 & 3.6 & 4.7 & 4.7 & 8.9 & 6.1 & 2.3 & 6.0 & 5.9 & 4.7 \\
\hline $\mathrm{LBP}^{\# M m \Sigma}$ & 86.0 & 70.6 & 67.9 & 74.8 & 94.1 & 87.6 & 84.5 & 88.7 & 97.1 & 90.2 & 86.4 & 91.2 \\
\hline Gain & 1.2 & 5.1 & 4.2 & 3.5 & 4.7 & 5.3 & 9.3 & 6.4 & 2.0 & 5.2 & 5.6 & 4.2 \\
\hline
\end{tabular}

Table 4. Comparison between $\mathrm{LBP}^{\text {riu } 2}$ and the basic $\mathrm{LBP}^{\mathcal{A}}$ on Outex dataset.

- Using 3 or 4 attributes further improves the results, except when $P=24$. This can be explained by the fact that in this case, the number of labels is too high, which makes the histogram too sparse for the $\chi^{2}$ distance.

In addition, Table 5 presents a comparison between $\mathrm{LBP}_{P, R}^{r i u 2+\mathcal{A}}$ and $\mathrm{LBP}_{P, R}^{r i u 2}$, when $\mathcal{A}$ is mono attribute or $\{M m\}$. It can be seen that the performance of $\mathrm{LBP}_{P, R}^{r i u 2+\mathcal{A}}$ is, in most cases, better than $\mathrm{LBP}_{P, R}^{\mathcal{A}}$ or $\operatorname{LBP}_{P, R}^{\text {riu2 } 2}$ alone.

\subsection{2nd experiment: $\operatorname{CLBP}_{P, R}^{\mathcal{A}}$}

Because, when the number of labels become too large $(P=24)$, the use of several attributes become really inefficient due to the very high dimension of feature vectors, in this experiment we consider only a combination of at most two attributes.

Table 6, 7. 8 present the results obtained by our methods $\mathrm{CLBP}_{P, R}^{\mathcal{A}}$ on the three datasets (Outex, CUReT and UIUC) in comparison with other LBP-based methods. From these tables, we can make the following remarks 


\begin{tabular}{|c|c|c|c|c|c|c|c|c|c|c|c|c|}
\hline \multirow[t]{2}{*}{ Method } & \multicolumn{4}{|c|}{$(\mathrm{P}, \mathrm{R})=(8,1)$} & \multicolumn{4}{|c|}{$(\mathrm{P}, \mathrm{R})=(16,2)$} & \multicolumn{4}{|c|}{$(\mathrm{P}, \mathrm{R})=(24,3)$} \\
\hline & TC10 & TC12t & TC12 & Mean & TC10 & TC12t & TC121 & Mean & TC10 & $\Gamma \mathrm{C} 12 \mathrm{t}$ & $\mathrm{TC} 12 \mathrm{~h}$ & Mean \\
\hline $\mathrm{LBP}^{\text {riu2 }} 2$ & 84.8 & 65.5 & 63.7 & 71.3 & 89.4 & 82.3 & 75.2 & 82.3 & 95.1 & 85.0 & 80.8 & 87.0 \\
\hline $\mathrm{LBP}^{\#}$ & 52.4 & 37.4 & 32.0 & 40.6 & 66.5 & 51.3 & 47.1 & 55.0 & 77.0 & 67.5 & 58.2 & 67.6 \\
\hline $\mathrm{LBP}^{r i}$ & 85.3 & 66.6 & 65.7 & 72.5 & 91.5 & 83.5 & 78.2 & 84.4 & 96.1 & 86.3 & 81.6 & 88.0 \\
\hline $\operatorname{Gain}_{r}$ & 0.5 & 1.1 & 2.0 & 1.3 & 2.1 & 1.2 & 3.0 & 2.1 & 1.0 & 1.3 & 0.8 & 1.0 \\
\hline Gain $_{\#}$ & 32.9 & 29.2 & 33.7 & 31.9 & 25.0 & 32.2 & 31.1 & 29.47 & 19.17 & 18.80 & 23.37 & 20.44 \\
\hline $\mathrm{LBP}^{M}$ & 83.6 & 67.1 & 64.4 & 71.7 & 87.6 & 82.2 & 78.9 & 82.9 & 95.9 & 88.1 & 86.4 & 90.1 \\
\hline $\mathrm{LBP}^{r}$ & 86.3 & 68.5 & 64.6 & 73.2 & 91.0 & 84.5 & 79.9 & 85.1 & 96.7 & 88.1 & 84.2 & 89.8 \\
\hline Gain & 1.5 & 3.0 & 0.9 & 1.9 & 1.6 & 2.2 & 4.7 & 2.8 & 1.6 & 3.1 & 3.4 & 2.8 \\
\hline $\operatorname{Gain}_{N}$ & 2.7 & 1.4 & 0.2 & 1.5 & 3.4 & 2.3 & 1.0 & 2.2 & 0.8 & - & - & - \\
\hline $\mathrm{LBP}^{m}$ & 84.8 & 64.5 & 62.2 & 69.9 & 91.0 & 82.9 & 77.0 & 83.7 & 96.5 & 86.2 & 80.4 & 87.7 \\
\hline $\mathrm{LBP}^{\text {riu } 2+m}$ & 85.1 & 66.7 & 65.3 & 72.4 & 92.2 & 85.1 & 80.3 & 85.9 & 97.5 & 89.1 & 84.6 & 90.0 \\
\hline Gain $_{r}$ & 0.3 & 1.2 & 1.6 & 1.1 & 2.8 & 2.8 & 5.1 & 3.6 & 2.4 & 4.1 & 3.8 & 3.0 \\
\hline $\operatorname{Gain}_{m}$ & 0.3 & 2.2 & 3.1 & 2.5 & 1.2 & 2.2 & 3.3 & 2.2 & 1.0 & 2.9 & 4.2 & 2.3 \\
\hline $\mathrm{LBP}^{\Sigma}$ & 82.9 & 65.0 & 63.2 & 70.4 & 88.7 & 82.6 & 77.4 & 82.9 & 91.3 & 83.8 & 82.7 & 86.0 \\
\hline $\mathrm{LBP}^{\text {riu } 2+\Sigma}$ & 86.1 & 68.1 & 65.9 & 73.4 & 90.1 & 83.7 & 77.1 & 83.6 & 96.0 & 86.3 & 82.8 & 88.4 \\
\hline Gain $_{\text {riu2 }}$ & 1.3 & 2.6 & 2.2 & 2.1 & 0.7 & 1.4 & 1.9 & 1.3 & 0.9 & 1.3 & 2.0 & 1.4 \\
\hline $\operatorname{Gain}_{\Sigma}$ & 3.2 & 3.1 & 2.7 & 3.0 & 1.4 & 1.1 & - & 0.7 & 4.7 & 2.5 & 0.1 & 2.4 \\
\hline $\mathrm{LBP}^{M m}$ & 85.6 & 66.8 & 63.6 & 72.0 & 92.5 & 85.4 & 82.4 & 86.8 & 98.1 & 92.2 & 87.2 & 92.5 \\
\hline $\mathrm{LBP}^{\text {riu2+ }}$ & 85.9 & 69.8 & 66.8 & 74.2 & 93.0 & 85.5 & 82.8 & 87.1 & 98.2 & 91.8 & 87.1 & 92.4 \\
\hline Gain $_{\text {riu2 }}$ & 1.1 & 4.3 & 1.1 & 2.9 & 3.6 & 3.2 & 7.6 & 4.8 & 3.1 & 6.8 & 6.3 & 5.4 \\
\hline $\operatorname{Gain}_{M m}$ & 0.3 & 3.0 & 3.2 & 2.2 & 0.5 & 0.1 & 0.4 & 0.3 & 0.1 & - & - & - \\
\hline
\end{tabular}

Table 5. Comparison between $\mathrm{LBP}^{\text {riu } 2}, \mathrm{LBP}^{\mathcal{A}}$ and the mixed $\mathrm{LBP}^{\text {riu } 2+\mathcal{A}}$ on Outex dataset when $\mathcal{A}$ is a mono attribute or $\{M m\}$.

- Except when $\mathcal{A}=\{\#\}, \mathrm{CLBP}_{P, R}^{\mathcal{A}}$ outperforms the previous methods in all configurations.

- Between mono attributes, $M$ is the best configuration. It means that $\mathrm{CLBP}^{M}$ outperforms CLBC 12] that is exactly $\mathrm{CLBP}^{\Sigma}$.

- The combination of two attributes still improves the performance of our descriptors. The improvement in relation with CLBP_S/M/C varies from $0.5 \%$ to $4.5 \%$ depending on test configurations.

In addition, Figure 6 presents the best results of one configuration $(M \#)$ in comparison with the best results of recent methods on Outex dataset: LBP ${ }^{\text {riu } 2}$ [2], LTP 7], DLBP + NGF [10], VZ-MR8 20], VZ-Joint 20], CLBP_S_M/C 11] and CLBP_CLBC [12. As can be seen from it, because the topology-related attributes bring more information than the typical mapping riu2, our best results in complementary scheme improve significantly with respect to CLBP.

\section{Conclusions}

We have proposed a versatile and efficient variant of LBP for texture description. The proposed framework extends existing rotation invariant LBP based coding, including riu 2 and LBC, while enhancing their expressiveness and improving their discrimination capability. The classification results on three recent texture datasets prove the relevance of our framework. Used in combination with the

\footnotetext{
${ }^{2} \mathrm{CLBP}_{3,24}^{M \Sigma}$ is not tested on this dataset.
} 


\begin{tabular}{|c|c|c|c|c|c|c|c|c|c|c|c|c|}
\hline \multirow[t]{2}{*}{ Method } & \multicolumn{4}{|c|}{$(\mathrm{P}, \mathrm{R})=(8,1)$} & \multicolumn{4}{|c|}{$(\mathrm{P}, \mathrm{R})=(16,2)$} & \multicolumn{4}{|c|}{$(\mathrm{P}, \mathrm{R})=(24,3)$} \\
\hline & TC10 & $\mathrm{TC} 12 \mathrm{t}$ & TC12 h & Mean & TC10 & TC12 $\mathrm{t}$ & ТC12 h & Mean & TC10 & $\mathrm{TC} 12 \mathrm{t}$ & TC12 h & Mean \\
\hline $\mathrm{LBP}^{\text {riu2 }} 2$ & 84.8 & 65.5 & 63.7 & 71.3 & 89.4 & 82.3 & 75.2 & 82.3 & 95.1 & 85.0 & 80.8 & 87.0 \\
\hline LTP 7 & 94.1 & 75.9 & 74.0 & 81.3 & 97.0 & 90.2 & 86.9 & 91.3 & 98.2 & 93.6 & 89.4 & 93.8 \\
\hline DLBP & & & & & 97.7 & 92.1 & 88.7 & 92.8 & 98.1 & 91.6 & 87.4 & 92.4 \\
\hline $\mathrm{DLBP}+\mathrm{NGF}$ 10 & & & & & 99.1 & 93.2 & 90.4 & 94.2 & 98.2 & 91.6 & 87.4 & 92.4 \\
\hline CLBP_S_M/C & 94.5 & 81.9 & 82.5 & 86.3 & 98.0 & 91.0 & 91.1 & 93.4 & 98.3 & 94.0 & 92.4 & 94.9 \\
\hline CLBP_S/M $11=$ & 94.7 & 82.7 & 83.1 & 86.8 & 97.9 & 90.5 & 91.1 & 93.2 & 99.3 & 93.6 & 93.3 & 95.4 \\
\hline CLBP_S/M/C 11 & 96.6 & 90.3 & 92.3 & 93.0 & 98.7 & 93.5 & 93.9 & 95.4 & 98.9 & 95.3 & 94.5 & 96.3 \\
\hline \multicolumn{13}{|c|}{ Our proposed descriptors } \\
\hline $\mathrm{CLBP}^{\#}$ & 85.4 & 71.7 & 70.7 & 75.9 & 90.6 & 83.4 & 80.9 & 85.0 & 89.0 & 81.3 & 79.3 & 83.2 \\
\hline $\mathrm{CLBP}^{M}$ & 96.5 & 90.9 & 93.0 & 93.4 & 98.4 & 95.5 & 96.2 & 96.7 & 99.1 & 97.2 & 96.8 & 97.7 \\
\hline $\mathrm{CLBP}^{m}$ & 96.7 & 90.5 & 91.6 & 92.9 & 99.0 & 95.6 & 94.9 & 90.5 & 99.5 & 96.7 & 96.0 & 97.4 \\
\hline $\mathrm{CLBP}^{\Sigma}$ & 97.2 & 89.8 & 92.9 & 93.3 & 98.5 & 93.3 & 94.1 & 95.3 & 98.8 & 94.0 & 95.4 & 96.1 \\
\hline $\mathrm{CLBP}^{M \#}$ & 96.2 & 90.6 & 93.5 & 93.5 & 98.9 & 95.5 & 95.8 & 96.8 & 99.5 & 97.0 & 96.3 & 97.6 \\
\hline $\mathrm{CLBP}^{m \#}$ & 96.3 & 90.4 & 92.2 & 93.0 & 98.9 & 95.3 & 95.1 & 96.4 & 99.3 & 96.4 & 96.0 & 97.2 \\
\hline $\mathrm{CLBP}^{M \Sigma}$ & 96.3 & 91.1 & 93.4 & 93.6 & 98.9 & 95.3 & 96.5 & 96.9 & 99.4 & 94.3 & 93.4 & 95.7 \\
\hline $\mathrm{CLBP}^{M m}$ & 96.8 & 90.9 & 93.2 & 93.6 & 98.9 & 95.7 & 95.2 & 96.6 & 99.3 & 95.5 & 93.8 & 96.2 \\
\hline $\mathrm{CLBP}^{\# \Sigma}$ & 96.7 & 90.7 & 93.6 & 93.6 & \begin{tabular}{|l|}
99.0 \\
\end{tabular} & 95.4 & 96.1 & 96.8 & 99.6 & 96.2 & 95.8 & 97.2 \\
\hline $\mathrm{CLBP}^{m \Sigma}$ & 96.5 & 90.6 & 92.3 & 93.1 & 99.0 & 95.23 & 96.1 & 96.8 & 99.1 & 94.6 & 93.0 & 95.5 \\
\hline
\end{tabular}

Table 6. Comparison between $\operatorname{CLBP}_{P, R}^{\mathcal{A}}$ and other LBP-based methods on Outex dataset.

complemented LBP coding, it even outperforms the state-of-the art LBP based descriptors. In the future, we plan to address the problem of high dimensionality when using more attributes in complemented LBPs.

\section{References}

1. T. Ojala, M. Pietikäinen, and D. Harwood, "A comparative study of texture measures with classification based on featured distributions," Pattern Recognition, vol. 29(1), pp. 51-59, 1996.

2. T. Ojala, M. Pietikäinen, and T. Mäenpää, "Multiresolution gray-scale and rotation invariant texture classification with local binary patterns," IEEE Trans. PAMI, vol. 24, pp. 971-987, 2002.

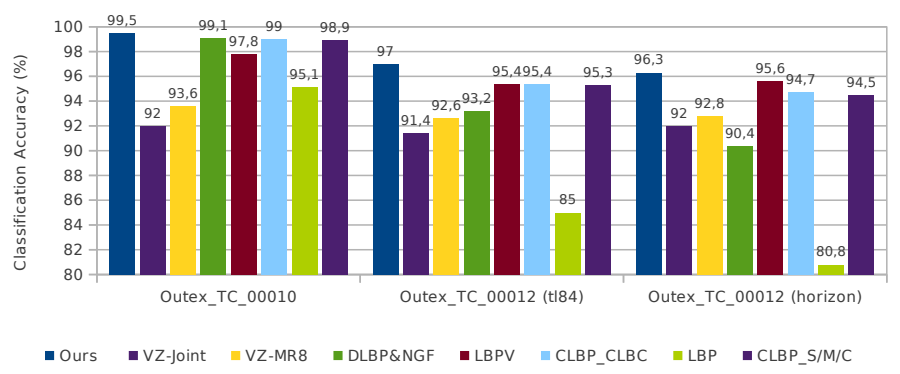

Fig. 6. Comparing the best results of $\mathrm{CLBP}^{M \#}$ with the best results of recent methods on Outex dataset. 


\begin{tabular}{|c|c|c|c|c|c|c|c|c|c|c|c|c|}
\hline \multirow[t]{2}{*}{ Method } & \multicolumn{4}{|c|}{$(\mathrm{P}, \mathrm{R})=(8,1)$} & \multicolumn{4}{|c|}{$(\mathrm{P}, \mathrm{R})=(16,3)$} & \multicolumn{4}{|c|}{$(\mathrm{P}, \mathrm{R})=(24,5)$} \\
\hline & $\mathrm{N}=46$ & $\mathrm{~N}=23$ & $\mathrm{~N}=12$ & $\mathrm{~N}=6$ & $\mathrm{~N}=46$ & $\mathrm{~N}=23$ & $\mathrm{~N}=12$ & $\mathrm{~N}=6$ & $\mathrm{~N}=46$ & $\mathrm{~N}=23$ & $\mathrm{~N}=12$ & $\mathrm{~N}=6$ \\
\hline LTP 7 & 85.13 & 79.25 & 72.25 & 63.09 & 92.66 & 87.30 & 80.22 & 70.50 & 91.81 & 85.78 & 77.88 & 67.77 \\
\hline $\mathrm{LBP}^{\text {riu } 2} / \mathrm{VA} \overline{\mathrm{R}}_{P, R} 21$ & 93.87 & 88.76 & 81.59 & 71.03 & 94.20 & 89.12 & 81.64 & 71.81 & 91.87 & 85.58 & 77.13 & 66.04 \\
\hline CLBP S/M/C 11 & 95.6 & 91.3 & 84.9 & 74.8 & 95.9 & 92.1 & 86.1 & 77.0 & 94.7 & 90.3 & 83.8 & 74.5 \\
\hline CLBP S/M $1 \overline{1}$ & 93.5 & 88.7 & 81.9 & 72.3 & 94.4 & 90.4 & 84.2 & 75.4 & 93.6 & 89.1 & 82.5 & 73.3 \\
\hline \multicolumn{13}{|c|}{ Our proposed descriptors } \\
\hline CLBP \# & 81.8 & 72.2 & 61.8 & 52.0 & 82.3 & 72.8 & 61.6 & 50.2 & 77.7 & 67.6 & 56.5 & 45.2 \\
\hline $\mathrm{CLBP}^{M}$ & 95.7 & 91.5 & 84.1 & 74.0 & 96.1 & 92.5 & 85.7 & 76.3 & 96.4 & 92.3 & 85.9 & 77.6 \\
\hline $\mathrm{CLBP}^{m}$ & 95.8 & 91.3 & 83.8 & 73.7 & 96.8 & 92.5 & 85.8 & \begin{tabular}{|l|}
77.1 \\
\end{tabular} & 95.4 & 91.6 & 85.4 & 77.8 \\
\hline $\mathrm{CLBP}^{\Sigma}$ & 94.8 & 90.1 & 82.7 & 72.1 & 94.7 & 89.8 & 82.3 & \begin{tabular}{|l|}
72.0 \\
\end{tabular} & 93.9 & 87.5 & 80.6 & 69.0 \\
\hline $\mathrm{CLBP}^{m \#}$ & 95.9 & 91.7 & 84.4 & 74.5 & 97.0 & 93.2 & 86.7 & 78.2 & 96.1 & 92.6 & 85.9 & 78.6 \\
\hline $\mathrm{CLBP}^{M \Sigma}$ & 96.3 & 92.1 & 84.8 & 74.8 & 96.0 & 92.0 & 85.7 & \begin{tabular}{|l|}
76.5 \\
\end{tabular} & $\mathrm{x}$ & $\mathrm{x}$ & $\mathrm{x}$ & $\mathrm{x}$ \\
\hline $\mathrm{CLBP}^{M m}$ & 96.2 & 91.9 & 84.7 & 74.6 & 96.7 & 93.1 & 86.7 & 78.1 & 94.5 & 90.45 & 83.8 & 77.0 \\
\hline $\mathrm{CLBP}^{\# \Sigma}$ & 96.2 & 91.8 & 84.6 & \begin{tabular}{|l|}
74.8 \\
\end{tabular} & 96.5 & 92.7 & 86.2 & \begin{tabular}{|l|}
77.1 \\
\end{tabular} & 93.7 & 89.0 & 81.5 & 73.8 \\
\hline $\mathrm{CLBP}^{m \Sigma}$ & 96.3 & 91.9 & 84.9 & 74.4 & 96.7 & 92.5 & 86.7 & 77.1 & 92.27 & 88.1 & 80.6 & 73.7 \\
\hline $\mathrm{CLBP}^{M \#}$ & 96.3 & 92.1 & 84.7 & 75.1 & 96.8 & 93.2 & 87.3 & 78.2 & 95.01 & 91.2 & 84.24 & 77.1 \\
\hline
\end{tabular}

Table 7. Experimentation of $\mathrm{CLBP}_{P, R}^{\mathcal{A}}$ on CUReT dataset ${ }^{2}$

3. Pietikinen Matti, Hadid Abdenour, Zhao Guoying, and Ahonen Timo, Computer Vision Using Local Binary Patterns, 2011.

4. Wenchao Zhang, Shiguang Shan, Wen Gao, Xilin Chen, and Hongming Zhang, "Local Gabor Binary Pattern Histogram Sequence (LGBPHS): A Novel Non-Statistical Model for Face Representation and Recognition," in ICCV, 2005, pp. 786-791.

5. Shu Liao and Albert C. S. Chung, "Face recognition by using elongated local binary patterns with average maximum distance gradient magnitude," in $A C C V$, Yasushi Yagi, Sing Bing Kang, In-So Kweon, and Hongbin Zha, Eds., 2007, vol. 4844 of $L N C S$, pp. $672-679$.

6. L. Wolf, T. Hassner, and Y. Taigman, "Descriptor based methods in the wild," in Real-Life Images Workshop, ECCV, 2008.

7. Xiaoyang Tan and Bill Triggs, "Enhanced local texture feature sets for face recognition under difficult lighting conditions," IEEE Trans. Image Processing, vol. 19, no. 6, pp. 1635-1650, 2010.

8. Mäenpää T. and Pietikäinen M., "Multi-scale binary patterns for texture analysis," in SCIA, 2003, vol. 2749 of $L N C S$, pp. 885-892.

9. S.C. Liao, X.X. Zhu, Z. Lei, L. Zhang, and S.Z. Li, "Learning multi-scale block local binary patterns for face recognition," in $I C B$, 2007, vol. 4642 of $L N C S$, pp. 828-837.

10. S. Liao, M. W. K. Law, and A. C. S. Chung, "Dominant local binary patterns for texture classification," IEEE Trans. Image Processing, vol. 18, no. 5, pp. $1107-$ 1118, May 2009.

11. Guo Z., Zhang Z., and Zhang D., "A completed modeling of local binary pattern operator for texture classification," IEEE Trans. Image Processing, vol. 19(6), pp. 1657-1663, 2010.

12. Yang Zhao, De-Shuang Huang, and Wei Jia, "Completed local binary count for rotation invariant texture classification," IEEE Trans. Image Processing, vol. 21, no. 10, pp. 4492-4497, 2012.

13. Shigeki Yokoi, Jun-Ichiro Toriwaki, and Teruo Fukumura, "An analysis of topological properties of digitized binary pictures using local features," CGIP, vol. 4, pp. 63-73, 1975. 


\begin{tabular}{|c|c|c|c|c|c|c|c|c|c|c|c|c|}
\hline \multirow[t]{2}{*}{ Method } & \multicolumn{4}{|c|}{$(\mathrm{P}, \mathrm{R})=(8,1)$} & \multicolumn{4}{|c|}{$(\mathrm{P}, \mathrm{R})=(16,2)$} & \multicolumn{4}{|c|}{$(\mathrm{P}, \mathrm{R})=(24,3)$} \\
\hline & 20 & 15 & 10 & 5 & 20 & 15 & 10 & 5 & 20 & 15 & 10 & 5 \\
\hline $\mathrm{LBP}^{\text {riu2 }}$ & 54.6 & 52.9 & 47.1 & 39.7 & 61.3 & 56.4 & 51.2 & 42.7 & 64.0 & 60.0 & 54.2 & 44.6 \\
\hline CLBP_S/M 19 & 81.8 & 78.5 & 74.8 & 64.8 & 87.9 & 85.1 & 80.6 & 71.6 & 89.2 & 87.4 & 81.9 & 72.5 \\
\hline $\mathrm{CLBP}_{-} \mathrm{S} / \mathrm{M} / \mathrm{C}^{-19}$ & 87.6 & 85.7 & 82.6 & 75.0 & 91.0 & 89.4 & 86.3 & 78.6 & 91.2 & 89.2 & 85.9 & 78.0 \\
\hline $\operatorname{CRLBP}(\alpha=1) \quad 22$ & 86.9 & 85.7 & 82.2 & 73.9 & 92.9 & 91.8 & 88.1 & 82.0 & 93.3 & 92.0 & 89.5 & 81.9 \\
\hline Number of training images $\mathrm{N}=$ & \multicolumn{3}{|c|}{20} & \multicolumn{3}{|c|}{15} & \multicolumn{3}{|c|}{10} & \multicolumn{3}{|c|}{5} \\
\hline Xu et al. 23 & \multicolumn{3}{|c|}{93.8} & \multicolumn{3}{|c|}{91.3} & \multicolumn{3}{|c|}{89.7} & \multicolumn{3}{|c|}{83.3} \\
\hline \multicolumn{13}{|c|}{ Our proposed descriptors } \\
\hline CLBP $\#$ & 75.0 & 70.8 & 67.0 & 59.5 & 70.8 & 65.7 & 60.3 & 49.9 & 66.4 & 60.9 & 55.4 & 44.3 \\
\hline $\mathrm{CLBP}^{M}$ & 88.0 & 85.8 & 82.9 & 75.2 & 92.1 & 90.7 & 87.9 & 81.1 & 93.1 & 92.3 & 88.7 & 81.9 \\
\hline $\mathrm{CLBP}^{m}$ & 87.3 & 84.5 & 81.6 & 73.7 & 91.3 & 89.6 & 86.2 & 78.3 & 92.1 & 90.3 & 86.5 & 78.1 \\
\hline $\mathrm{CLBP}^{\Sigma}$ & 88.1 & 85.6 & 82.8 & 75.2 & 90.8 & 89.4 & 86.7 & 79.9 & 91.2 & \begin{tabular}{|l}
89.9 \\
\end{tabular} & 86.9 & 79.4 \\
\hline $\mathrm{CLBP}^{M \#}$ & 88.1 & 86.2 & 83.2 & 76.0 & 92.5 & 90.9 & 88.4 & 80.8 & 93.8 & 92.0 & 89.2 & 81.6 \\
\hline $\mathrm{CLBP}^{m \#}$ & 87.8 & 85.7 & 82.5 & 75.4 & 92.4 & 90.6 & 88.0 & 80.3 & 93.5 & 91.6 & 88.5 & 80.6 \\
\hline $\mathrm{CLBP}^{M \nu}$ & 88.2 & 86.4 & 83.6 & 76.3 & 93.0 & 91.7 & 89.2 & 82.2 & 94.2 & 92.7 & 90.0 & 82.7 \\
\hline $\mathrm{CLBP}^{M m}$ & 88.2 & 86.2 & 83.4 & 76.0 & 92.9 & 91.6 & 89.1 & 81.8 & 94.4 & 92.8 & 90.1 & 82.6 \\
\hline $\mathrm{CLBP}^{\# \Sigma}$ & 88.4 & 86.4 & 83.6 & 76.3 & 92.3 & 90.6 & 88.2 & 80.9 & 93.1 & 91.4 & 88.6 & 80.7 \\
\hline $\mathrm{CLBP}^{m \Sigma}$ & 88.2 & 86.4 & 83.4 & 76.3 & 93.0 & 91.4 & 88.8 & 81.9 & 94.3 & 92.6 & 89.9 & 82.8 \\
\hline
\end{tabular}

Table 8. Experimentation of $\mathrm{CLBP}_{P, R}^{\mathcal{A}}$ on UIUC dataset.

14. Abdolhossein Fathi and Ahmad Reza Naghsh-Nilchi, "Noise tolerant local binary pattern operator for efficient texture analysis," Pattern Recognition Letters, vol. 33, no. 9, pp. 1093-1100, 2012.

15. Timo Ojala, Topi Menp, Matti Pietikinen, Jaakko Viertola, Juha Kyllnen, and Sami Huovinen, "Outex - new framework for empirical evaluation of texture analysis algorithms," in ICPR, 2002, pp. 701-706.

16. Kristin J. Dana, Bram van Ginneken, Shree K. Nayar, and Jan J. Koenderink, "Reflectance and texture of real-world surfaces," ACM Trans. Graph., vol. 18, pp. 1-34, 1999.

17. S. Lazebnik, C. Schmid, and J. Ponce, "A sparse texture representation using local affine regions," IEEE Trans. PAMI, vol. 27, no. 8, pp. 1265-1278, 2005.

18. Manik Varma and Andrew Zisserman, "A statistical approach to material classification using image patch exemplars," IEEE Trans. PAMI, vol. 31, no. 11, pp. 2032-2047, 2009.

19. Zhenhua Guo, Lei Zhang, and D. Zhang, "A completed modeling of local binary pattern operator for texture classification," IEEE Trans. Image Processing, vol. 19, no. 6, pp. 1657-1663, June 2010.

20. Manik Varma and Andrew Zisserman, "A statistical approach to texture classification from single images," International Journal of Computer Vision, vol. 62, no. 1-2, pp. 61-81, 2005.

21. T. Ojala, M. Pietikainen, and T. Maenpaa, "Multiresolution gray-scale and rotation invariant texture classification with local binary patterns," IEEE Trans. PAMI, vol. 24, no. 7, pp. 971-987, July 2002.

22. Yang Zhao, Wei Jia, Rong-Xiang Hu, and Hai Min, "Completed robust local binary pattern for texture classification," Neurocomputing, vol. 106, pp. 68-76, 2013.

23. Yong $\mathrm{Xu}, \mathrm{Hui} \mathrm{Ji}$, and Cornelia Fermüller, "Viewpoint invariant texture description using fractal analysis," International Journal of Computer Vision, vol. 83, no. 1, pp. 85-100, 2009. 